\title{
Analytical Solution for the Free Over-Fall Weir Flow Using Conformal Mapping and Potential Flow Theory
}

\author{
A. R. Kabiri-Samani*, M. Amirabdollahian, F. Farshi \\ Department of Civil Engin eering, Isfahan University of Technology, Isfahan, 84156-83111, Iran
}

\begin{abstract}
In this study an analytical approach is presented based on the potential flow theory and conformal mapping technique to solve the problem of an ideal and steady flow over a free over-fall weir. The results are arranged for rectangular sharp-crested over-fall weirs with different vertical aspect ratios $(h / P, h$ is the water head above the weir and $P$ is the weir height). To validate the results of this approach, the free over-fall weir $d$ ischarge equation and the water free-surface profiles obtained with the potential flow theory have been calibrated with the experimental data. In conclusion, the information made available and the close correlation among the results of present analytical ap proach and the experimental data are adequate to warrant the recommendation of this method as a valuable supplement to existing methods employed in engineering design.
\end{abstract}

Keywords Free Over-Fall Weir, Potential Flow, Conformal Mapping, Schwarz-Christoffel Transformation, Discharge Coefficient, Free-Surface Profile

\section{Introduction}

Weirs are simple and precise structures for flow measurement and control in open channels. Formerly, the weir equations were found mainly on the basis of experimental data. Based on the prevailing universal weir discharge equation, the discharge over the rectangular sharp-crested weirs could be revealed as [1]

$$
q=\frac{2}{3} C_{d} \sqrt{2 g} h^{1.5}
$$

where $q$ is the discharge per unite length of the weir crest, $h$ is the upstream water head, $g$ is the gravitational acceleration and $C_{d}$ is the weir discharge coefficient. Formerly, the weir discharge coefficient was determined mainly on the bas is of experimental data and empirical equations. The flow characteristics including the discharge coefficient, velocity and pressure distribution and water surface profiles over the weirs were the subject of many investigations [2-9]. The problem studied here is concerned with the 2-D, steady, irrotational and free-surface flow. The fluid could be assumed as incompressible and inviscid. Hence, this nonlinear problem could be solved by various approaches. An alternative approach is employing the potential flow theory to determine the flow pattern i.e. velocity potential and stream functions through passing the weirs. The determination of the flow pattern in the vicinity of a weir has many applications, such as the determination of the

* Corresponding author:

akabiri@cc.iut.ac.ir (A. R. Kabiri-Samani)

Published online at http://journal.sapub.org/ijhe

Copyright (C) 2012 Scientific \& Academic Publishing. All Rights Reserved discharge coefficient, the calculation of the pressure force exerted over the weir and determination of the distortion of the velocity profile induced by flow contraction. Potential theory allows one to calcu late explicitly the free streamlines past the weir. For the external flows above the bodies such as weirs, potential flow theory should work well, until the surface pressure gradient becomes adverse. Having found velocity potential $(\varphi)$ and the equipotential lines or stream function along the flow lines from such analysis, the flow velocity and discharge coefficient would be computed by direct differentiation of potential function. From the mathematical viewpoint, the direct calculation of free streamlined flows is restricted to two-dimensional, irrotational flows of incompressible fluids for which the solid boundaries are straight. As these strict limitations are seldom fully satisfied by real flu id fields, the practical value of the results is open to question and relates to the problem which is solved. The significance of the various restrictions can be shown only by comparing the calculated results with those obtained from observations or real data. Afzalimehr and Bagheri[10] developed an equation for estimating the discharge coefficient of sharp-crested weirs based on potential flow theory. They compared the results with the experimental data and suggested an equation for the discharge coefficient for a large range of vertical aspect ratios.

Potential flow theory leads to the analytic determination of flow characteristics based on a transformation between the physical plane and the complex potential plane using conformal mapping technique. Conformal mapping is a mathematical technique in which complicated geometries can be transformed by a mapping function into simpler 
geometries which still preserves both the angles and orientation of the original geometry[11]. Conformal mapping transformations are both mathematically interesting and practically useful[12-17].

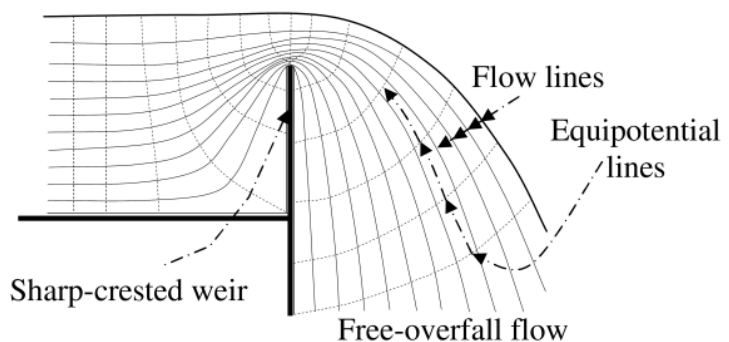

Figure 1. Streamlines and equipotential lines for flow over a free over-fall weir

Although ample studies have been carried out to investigate the flow characteristics passing sharp-crested weirs but limited information is available reported in the published literature on the free over-fall weir flow characteristics. Free over-fall we ir includes a sharp-crested weir installed above a deep drop aimed to force falling streamlines to be in vertical orientation (Figure 1). In this paper, the study focuses on modeling the 2-D flu id flow over the free over-fall weirs using the conformal mapping technique. The Schwarz-Christoffel transformation, a specific application of conformal mapping, was used to achieve the solution for flow over free over-fall weirs. The solution of potential flow for an over-fall weir will be an orthogonal flow net as shown in Figure 1. The application of computational tools to implement this conformal mapping transformation in order to compute the flow field, water surface profile and weir discharge equation is discussed. Finally, the results of analytical modeling were compared with the experimental data of the present study. These results will be supplemented with a quantitative error analys is in an attempt to explain any discrepancies among the models.

\section{Theoretical Considerations}

In complex systems, a Sch warz-Christoffel technique is a conformal transformation of a simple polygon interior area onto the upper half-plane $\operatorname{Im}(z)>0$, which is also used in potential flow theory and some of its applications. Suppose a polygon in the $w$-plane with vertices at $w_{1}, w_{2}, \ldots, w_{\mathrm{n}}$ and interior angles of $\alpha_{1}, \alpha_{2}, \ldots, \alpha_{\mathrm{n}}$. Where these points map onto points $x_{1}, x_{2}, \ldots, x_{\mathrm{n}}$ on the real axis of the $z$-plane (Figure 2 ). Let $G$ denote the interior of the polygon and $D$ the interior of the upper half-plane. Then a transformation $w=F(z)$ maps $D$ onto $G$ is given by the Schwarz-Christoffel transformation as

$$
\begin{aligned}
& F(z) \\
= & \left(\int A\left(z-x_{1}\right)^{-\alpha_{1} / \pi}\left(z-x_{2}\right)^{-\alpha_{2} / \pi} \ldots . .\left(z-x_{n}\right)^{-\alpha_{n} / \pi}\right)+B^{(2)}
\end{aligned}
$$

where the constants $A$ and $B$ demonstrate the size and position of $G$ and $x_{1}, x_{2}, \ldots, x_{\mathrm{n}}$ are the real part of complex variable $z(=x+i y)$ in the $z$-plane[11]. The complex potential $w=F(z)$ is a conformal transformation which maps the stream function and velocity potential curves in $w$-plane (physical plane) to a set of horizontal and vertical lines in $z$-plane (complex plane). Hence, the main purpose of the analysis is to survey the appropriate form of complex potential $F(z)$, which converts the complicated flow field to parallel sets of horizontal and vertical lines [18]

Applying the potential flow theorem and conformal mapping procedure to discover the image of a flow passing an over-fall weir, two points $x_{k}$ might be chosen arbitrarily in $z$-plane corres ponding to the comple $\mathrm{x}$ variables $w_{k}$ in $w$-plane as (Figure 3):

$$
\begin{aligned}
& x_{1}=0 ; w_{1}=0 ; \alpha_{1}=\pi / 2 \\
& x_{2}=P ; w_{2}=i P ; \alpha_{2}=-\pi
\end{aligned}
$$

Substituting the chosen $x_{k}$ and $\alpha_{k}(k=1,2)$ into Eq.(2), yields to the following integration for complex potential $F(z)$ :

$$
\begin{gathered}
F(z)=\left(\int A(z-0)^{-(\pi / 2) / \pi}(z-P)^{-(-\pi) / \pi}\right)+B \\
F(z)=A\left(\frac{2}{3} z^{3 / 2}-2 P z^{1 / 2}\right)+B
\end{gathered}
$$
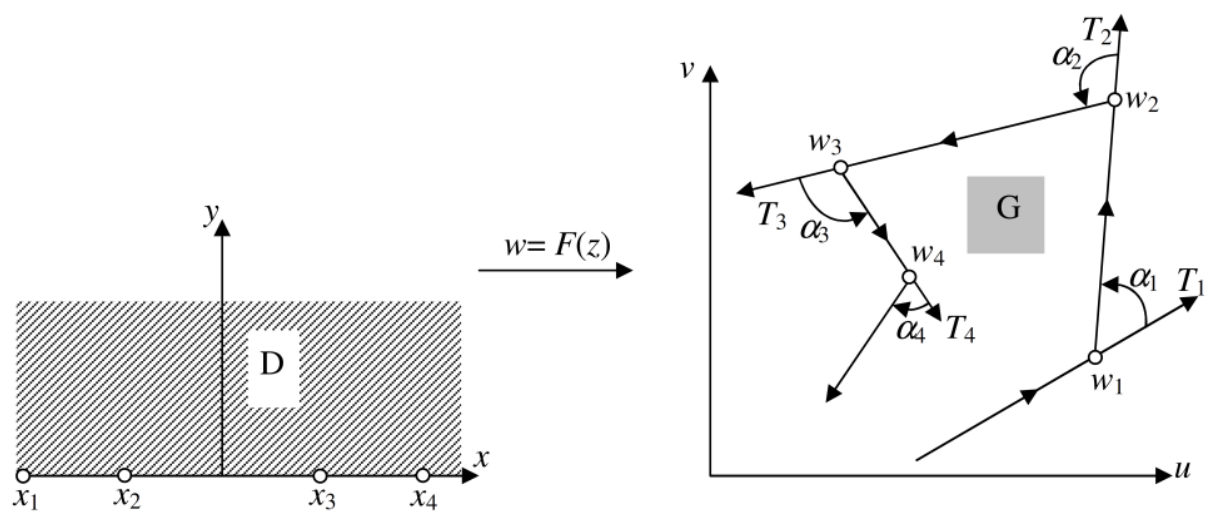

Figure 2. Conformal mapping based on Schwarz-Christoffeltransformation 


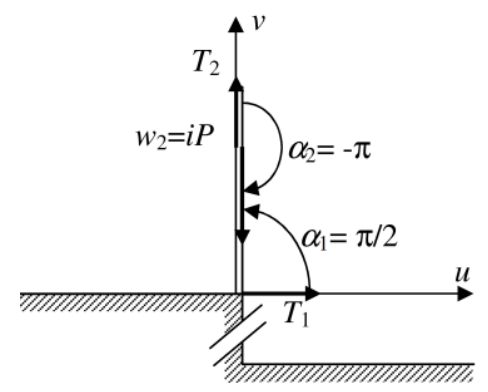

Figure 3. w-plane, the complex potential plane corresponding to flow over an over-fall weir

Consequence to integrating Eq.(4) and applying the conditions of $F(0)=0, F(P)=i P$, constants $A$ and $B$ are calculated, hence the comple x potential $F(z)$ could be written as:

$$
F(z)=\left(i \frac{-3}{4 P^{0.5}}\right)\left(\frac{2}{3} z^{3 / 2}-2 P z^{1 / 2}\right)
$$

Since $z=r \mathrm{e}^{i \theta}, F(z)=F(r, \theta)$ could be expressed as:

$$
F(z)=\left(i \frac{-3}{4 P^{0.5}}\right)\left(\frac{2}{3} r^{3 / 2}\left(\operatorname{Cos}\left(\frac{3 \theta}{2}\right)+i \operatorname{Sin}\left(\frac{3 \theta}{2}\right)\right)-2 \operatorname{Pr}^{1 / 2}\left(\operatorname{Cos}\left(\frac{\theta}{2}\right)+i \operatorname{Sin}\left(\frac{\theta}{2}\right)\right)\right)
$$

To illustrate the interest of the approach, the equipotential and streamlines for different configurations in order to analyze the effects of the boundaries on the flow field were calculated. Afterward, the study focuses on the configuration and hydrodynamic aspects of the flow through over-fall weir and compares the solution with experimental data. While $F(z)$ $=w=u+i v, u$ and $v$ could be expressed as the real and imaginary parts of Eq.(7), respectively. Since $F(z)$ just involves the geometries of the problem and the hydraulic aspects are not included, $u$ and $v$ are representatives of velocity potential and flow stream functions (e.g. $x$ and $y$ respectively). By deriving the real and imaginary parts of Eq.(7) an arbitrary point on orthogonal lines in the complex plane, is mapped to corresponding point $(u, v)$ in physical plane. Defining the streamlines passing a free over-fall weir with the height $P$, the horizontal lines in $z$-plane are mapped to the physical plane of $w$ using Eq.(7). As the derived equation is very complicated and could not straightforwardly be separated to achieve the real and imag inary parts, hence a numerical computation was applied using Mathematica software. Based on the above procedure the pair of $(u, v)$ were achieved for particular $x$ and $y$ values. Figures 4 and 5 show typical representatives of flow streamlines and velocity potential curves respectively. The weir height was considered to be $1.5 \mathrm{~m}$.

Besides, different figures were plotted to illustrate the effects of boundaries on the flow field. The weir contours and the bottom consist of solid constraints in the motion. The flow is forced by the gravity and viscosity and surface tension effects are neglected. Results indicated that, in general in the close vicinity of the weir crest, the streamlines deviate significantly. As expected, at a larger distance from the weir (about $x / P \geq 5$ ), less distortion of the equipotential lines is observed and the water free-surface is nearly horizontal. This result was proved by different investigators [9].

\section{Model Experimentation}

To investigate the water free-surface profile and over-fall weir discharge equations, experimental study was performed in a rectangular glass open channel of $11 \mathrm{~m}$ length, $0.4 \mathrm{~m}$ width and $1.0 \mathrm{~m}$ height. Experimental setup consists of a circulating system including main channel, pump, upstream and downstream reservoirs, etc. The longitudinal slope of the channel was set to be zero. Free over-fall weirs were made of $3 \mathrm{~mm}$ thick steel sheets with sharp edged and fully aerated condition. Two weirs with $P=5$ and $10 \mathrm{~cm}$ were considered and placed at the mid-length of the upstream in let above the corner of a $0.4 \mathrm{~m}$ high vertical drop. Series of tests were conducted for different flow conditions and each corresponding inlet discharge $(Q)$ was measured using an ultrasound flow-meter with a precision of $\pm 0.1 \mathrm{lit} / \mathrm{s}$. The minimum of eight discharges between 20 and 60 lit/s were considered. To evaluate the velocity profiles aimed to investigate the flow structure at the test section, the 3-D velocity profiles were measured using an acoustic doppler velocimeter (ADV). The depths of flow along and across the channel and over the crest of the weirs were measured using a point gauge mounted on a movable beam with a precision of $\pm 0.1 \mathrm{~mm}$. Experiments were conducted for sub-critical, stable and free over-fall conditions. 


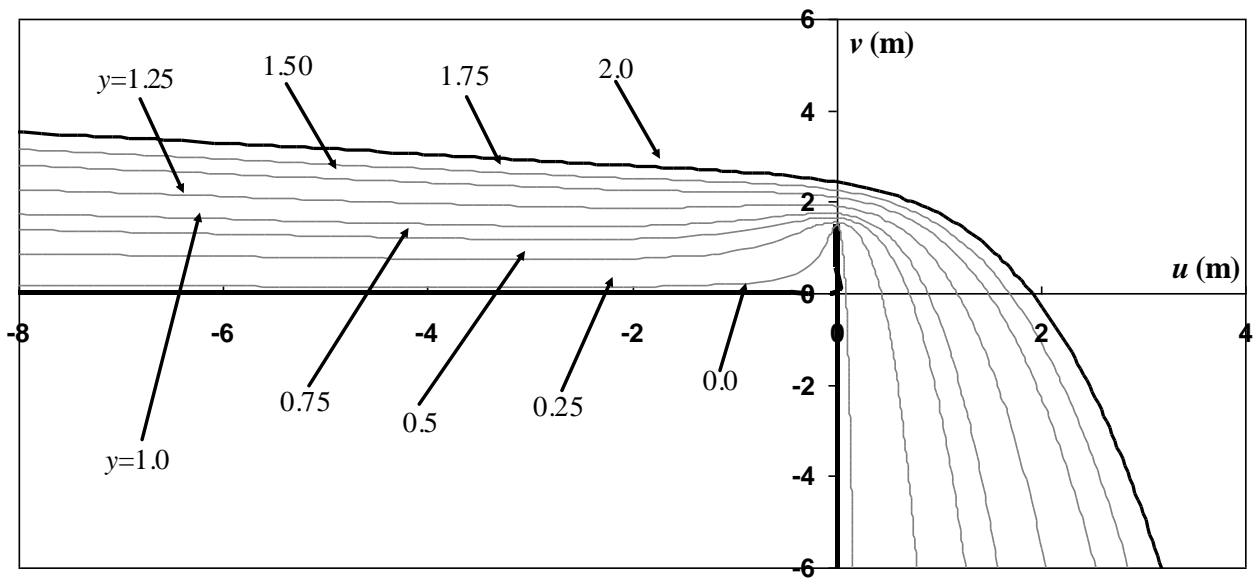

Figure 4. Typical calculated streamlines over an over-fall weir with $P=1.5 \mathrm{~m}$

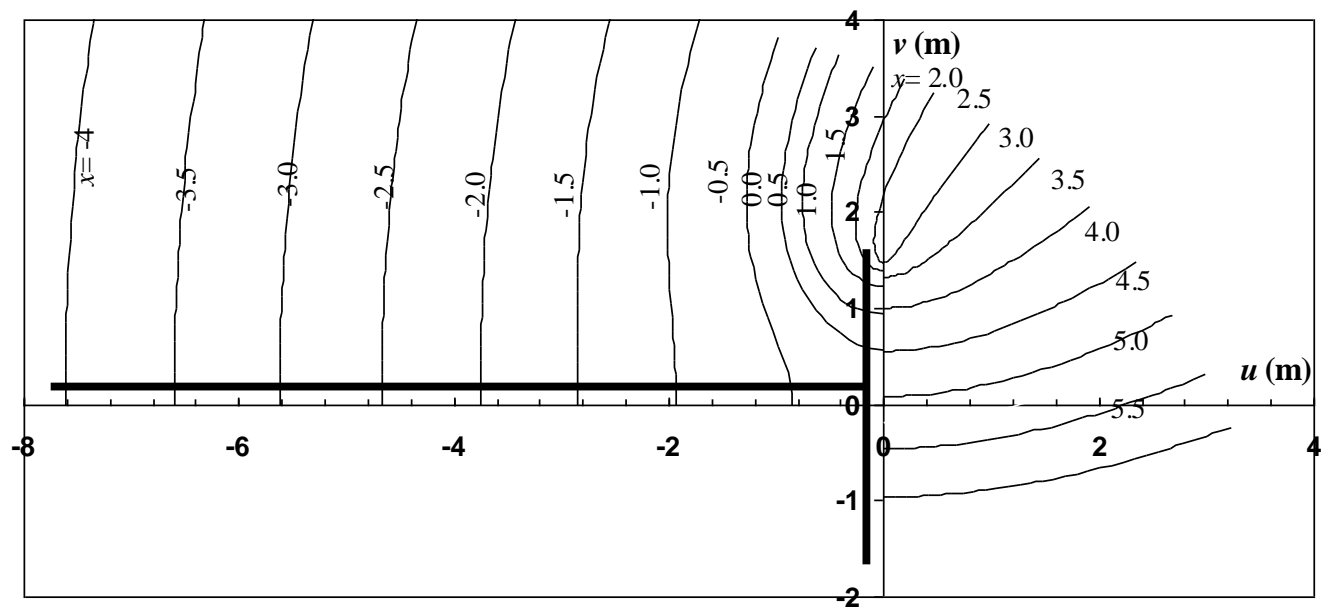

Figure 5. Potential velocity curves over an over-fall weir with $P=1.5 \mathrm{~m}$

\section{Results and Discussions}

\subsection{Water Surface Profile}

In this section the analytical results obtained applying the potential flow theory and conformal mapping technique, are presented. As mentioned before, with the advances in analytical methods, computational models of flow through hydraulic structures are increasingly being used in industry, but they still require validation from experiments. For this reason, the analytical results have been compared against experimental measurements of the present study. The laboratory measurements regard the water elevation above the over-fall weir in order to track the free-surface profile. Consequent to applying the proposed analytical model, the water free-surface profiles were calculated for particular over-fall weir geometry by transforming the horizontal line passing from the upstream normal water depth $(h)$ in complex plane, into physical domain using Eq.(7). Comparing the analytical results and experimental data indicated that, there were meaningful discrepancies between the analytical and experimental results of water free-surface. This is in part due to the simplifying assumptions of the potential flow. Hence, the theoretical results were improved based on experimental data and trial and error procedure using a quadratic correlation as follows:

$$
\left(\frac{v}{h}\right)_{a c t}=A\left(\frac{v}{h}\right)_{a n a}^{2}+B\left(\frac{v}{h}\right)_{a n a}+C
$$

where:

$$
\begin{aligned}
A= & 1.09(h / P)^{5}-9.41(h / P)^{4}+30.90(h / P)^{3} \\
& -48.06(h / P)^{2}+35.28(h / P)-9.88 \\
B= & -1.58(h / P)^{5}+13.50(h / P)^{4}-44.10(h / P)^{3} \\
& +68.21(h / P)^{2}-49.76(h / P)+14.20 \\
C= & 0.26(h / P)^{6}+3.57(h / P)^{5}-18.63(h / P)^{4} \\
- & 48.42(h / P)^{3}+66.17(h / P)^{2}+45.12(h / P)-11.43
\end{aligned}
$$

where $(y / h)_{\text {act }}$ and $(y / h)_{\text {ana }}$ are the actual non-dimensional vertical coordinate and the dimensionless vertical coordinate of the water free-surface obtained based on analytical approach respectively. No doubt due to different combinations, many functions can be introduced for each trial and error turn but, one's judgment can achieve more accurate results. 

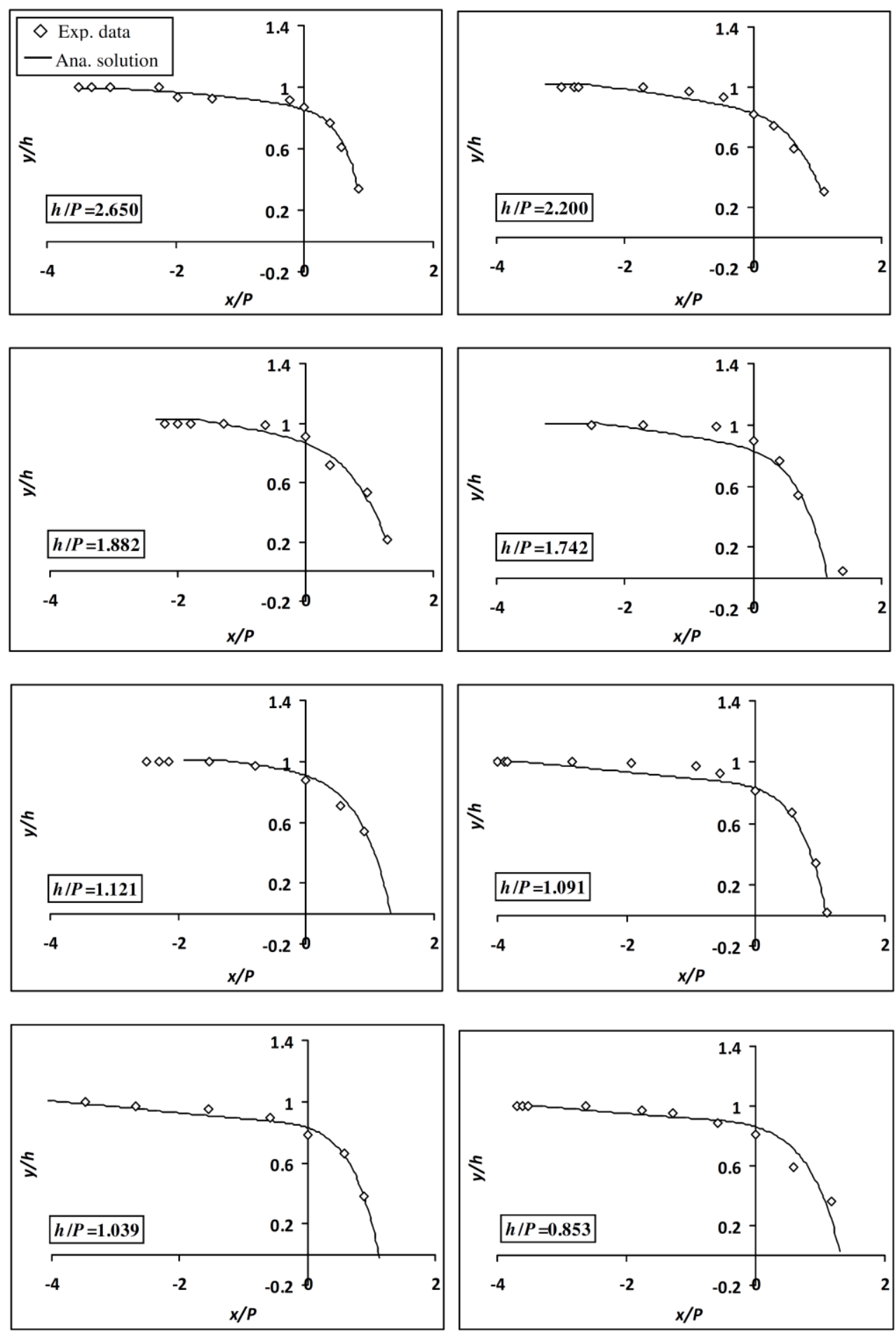

Figure 6. The analytical water free-surface profiles compared with the experimental dat a for different $h / P$ ratios

Figure 6 shows the predicted non-dimensional flow profiles denoting $y / h$ as a function of the non-dimensional distance $x / P$ for subcritical approach flows respect to different $h / P$ values. In Figure 6 , the improved surface profiles of the analytical modeling are compared with the experimental profiles. As illustrated, the present improved analytical profiles agree very well with the model experimentation results. Results signified that, the nappe downstream the weir has a contraction preserving the continuum free-surface profile and property like a jet.

To evaluate the validity of the achieved analytical free-surface results compared with the experimental data, the error functions normalized root mean square error (NRMSE) and weighted quadratic deviation (WQD) expressed by Eqs.(12) and (13) were used. The coefficient of determination $\left(\mathrm{R}^{2}\right)$ is also calculated. 


$$
\begin{gathered}
\text { NRMSE }=\sqrt{\frac{\sum(F(x)-f(x))^{2}}{\sum(F(x)-\bar{f})^{2}}} \\
W Q D=\sqrt{\frac{\sum\left(F(x) f(x)(F(x)-f(x))^{2}\right.}{\sum(F(x) f(x))}}
\end{gathered}
$$

where $f(x)$ and $f^{-}$are the experimental values and the average of the experimental data respectively and $F(x)$ is the estimated results. Contrary to $\mathrm{R}^{2}$, the values of NRMSE and WQD must be the smallest[19]. The reported NRMSE, WQD and $\mathrm{R}^{2}$ values in this analysis are $0.17,0.005$ and 0.97 , respectively, which show good agreement between theoretical and experimental results. Figure 7 shows the comparison between analytical normalized water free-surface profiles against the experimental data. In this figure, the majority of $\pm 7 \%$ bounds are also shown. As almost all the data points lie with in $\pm 7 \%$ tolerance, it means that the analytical model is in good agreement with the experimental results.

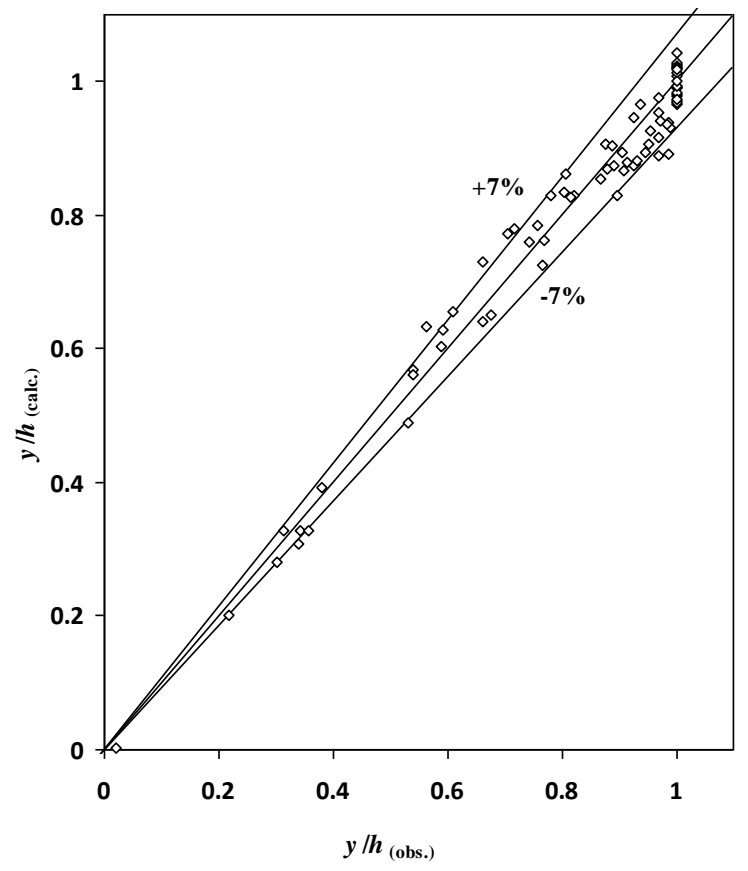

Figure 7. Experimental versus theoretical flow depths

\subsection{Discharge Coefficient}

The complex potential of flow field includes two parts, (i) a real part, indicating the velocity potential $(\varphi)$ and (ii) an imaginary part, which specifies the stream function $(\psi)$. According to Eq.(7) $x=$ cte and $y=c t e$ are analogies to $\varphi$ and $\psi$ functions, respectively. Based on numerical solution and applying nonlinear regression analysis the stream functions were expressed as follows:

$$
\begin{aligned}
y= & 6.358 v+8.746 P+49.546 u-\frac{11.546 P}{0.538+(u / v)^{2}} \\
& -85.764 v(u / P)+38.899 u(v / P)^{1.498} \\
& +6.282\left(P^{3} /\left(u^{2}+v^{2}\right)\right)=\text { Constant }
\end{aligned}
$$

then:

$$
\psi=k_{0} y U_{\infty}
$$

where $U_{\infty}$ and $k_{0}$ denote the ideal approaching flow velocity and correction factor taking into account the simplifications and errors of analysis. The coefficient of determination for Eq.(14) is about 0.97. Note that Eq.(14) is valid at the vicinity of the weir crest, where was aimed for discharge calculation. Since, $u=0$ over the weir crest, combining Eqs.(14) and (15) yields to the following expression:

$$
\psi=k_{0} U_{\infty}\left\{6.358 v+8.746 P+6.282\left(P^{3} / v^{2}\right)-\frac{11.546 P}{0.538}\right\}(16)
$$

By deriving Eq.(16), the flow velocity component and the flow discharge were calculated as follows:

$$
\begin{gathered}
U=\frac{\partial \psi}{\partial v}=k_{0} U_{\infty}\left\{6.358-12.564\left(P^{3} / v^{3}\right)\right\} \\
q=\int_{P}^{P+y_{p}} k_{0} U_{\infty}\left\{6.358-12.564\left(P^{3} / v^{3}\right)\right\} d v
\end{gathered}
$$

where $y_{P}$ and $q$ are the water depth exactly over the weir crest and discharge per unit length. Tacking into account the boundary conditions and simplifying of Eq.(18) yields to the following expression for estimating the flow discharge over the over-fall weirs.

$$
q=k_{0} U_{\infty}\left[6.358 y_{p}+6.283 P^{3}\left(\frac{1}{\left(P+y_{p}\right)^{2}}-\frac{1}{P^{2}}\right)\right]
$$

By assuming $U_{\infty}=q /(P+h)$, then $y_{P}$ would be determined based on a trial and error procedure. If the free jet flow theory is employed for flow passing an over-fall weir, it tends to an approximate re lation between $U_{\infty}$ and $h$ as follow:

$$
U_{\infty}=k_{1} \sqrt{2 g h}
$$

where $k_{1}$ is a correction factor. If Eqs.(19) and (20) were combined; the following expression for estimating the flow discharge would be achieved.

$$
q=C_{0}\left[6.358 y_{p}+6.283 P\left(\frac{1}{\left(1+y_{p} / P\right)^{2}}-1\right)\right] \sqrt{2 g h}
$$

$C_{0}=k_{0} k_{1}$ is calculated based on actual data. By comparing Eqs.(1) and (21), the free over-fall we ir discharge coefficient $C_{d}$ can be presented as follows:

$C_{d}$

$=\frac{3}{2} C_{0}\left[6.358\left(y_{p} / h\right)+6.283(P / h)\left(\frac{1}{\left[1+\left(y_{p} / h\right)(h / P)\right]^{2}}-1\right)\right]$

It should be remarked that, the potential flow theory is valid for irrotational, ideal flow conditions, where the viscous effects are negligible. Assuming ideal flow condition for the flow passing a solid object is not valid in a thin layer surrounding the solid boundary. Likewise, surrounding an over-fall weir, concentrated vortices are developed and the potential flow assumptions can not be established. Hence, due to the importance of viscous and inertia effects as well as vortices consequences and flow separation, head loss arise through the flow passage and consequently the flow discharge reduces compared with the ideal flow condition. The coefficient $C_{0}$ takes in to account these influences. The 
above equation has been worked out in this study to present a new accurate and practical equation for estimating the discharge coefficient of the free over-fall weir flow. By employing the experimental data of the present study, based on a nonlinear regression analysis $C_{0}$ is expressed as follows :

$$
\begin{aligned}
C_{0}= & 1.328+0.178(h / P)-0.026(h / P)^{2} \\
& -19.066 \frac{h / P}{13.353 h / P+0.43}-\frac{21.864}{-536.244+(h / P)^{45.188}}
\end{aligned}
$$

subject to $h / P \leq 2.65$. Figure 8 shows the variation of observed versus calculated $C_{0}$. In this figure the majority of $\pm 5 \%$ bonds are also attached. As is illustrated analytical results well compared with the experimental ones. The corresponding NRMSE, W QD and $\mathrm{R}^{2}$ values for the above relationship are $0.15,0.003$ and 0.98 , respectively.

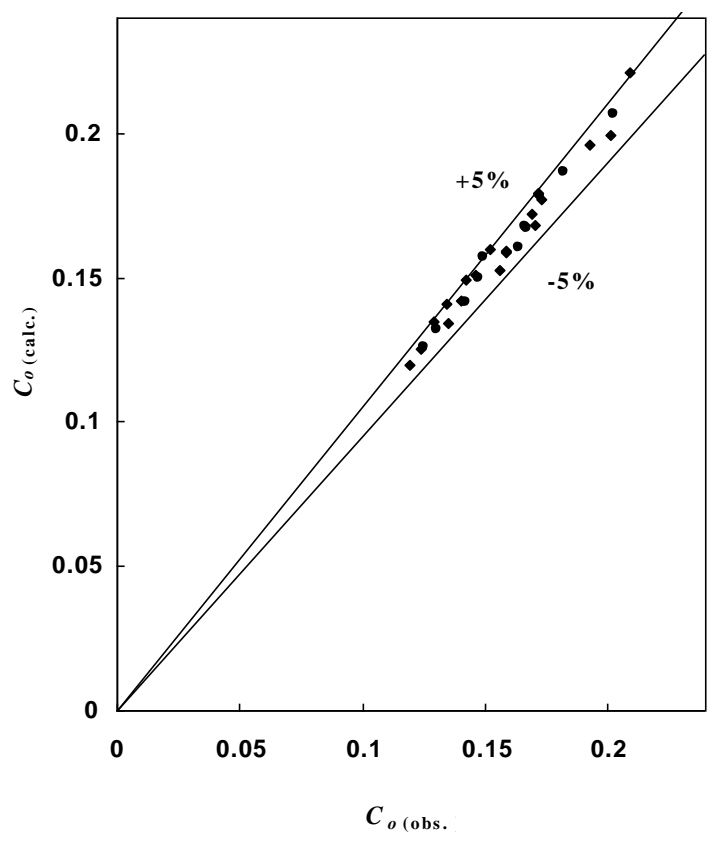

Figure 8. Comparison between calculated and observed $C_{0}$

\section{Conclusions}

The overall goal of this study was to effectively model the fluid flow over free over-fall weirs using the conformal mapping technique based on the potential flow theory. The solution for the inviscid flow over free over-fall weir has been mapped successfully using Schwarz Christoffel transformation. Consequent to employing the potential flow theory, equations for determining the water free-surface profile and the discharge coefficient of the free over-fall weirs were obtained. The analytical results were calibrated according to an experimental investigation. The predictions of the semi-analytical model agree well with the experimental data related to water surface profiles and discharge coefficient. The results reveal that the Schwarz-Christoffel is the most accurate transformation to solve such problems compared with the other ones. The results are validated for large rang of $h / P$ (i.e. $h / P \leq 2.65$, which are more practical in design considerations).

\section{REFERENCES}

[1] Bos, M.G. "Discharge measurement structures", International Institute for land Reclamation and Improvement, Wageningen NL (1989).

[2] Kandaswamy, P.K. and Rouse, H. "Characteristics of flow over terminal weirs and sills", Journal of Hydraulic Division, 83(4), pp. 1-13 (1957).

[3] Kindsvater, C.E. and Carter, R.W. "Discharge characteristics of rectangular thin plate", Journal of Hydraululic Division, 83(6), pp. 1-36 (1957).

[4] Rajaratnam, N. and Muralidhar, D. "Pressure and velocity distribution for sharp crest weirs", Journal of Hydraulic Research, 9(2), pp. 217-240 (1971).

[5] Hager, W.H. "Hydraulic of plane overfall", Journal of Hydraulic Engineering, 109(12) (1983) 1683-1697.

[6] Ramamurthy, A.S., Tim, U.S. and Rao, M.V.J. "Flow over sharp-crested plate weirs", Journal of Irrigation and Drainage Engineering, 113(2), pp. 163-172 (1987).

[7] Khan, A.A. and Steffler, P.M. "Modeling overfalls using vertically averaged and moment equations", Journal of Hydraulic Engineering, 122(7), pp. 397-402 (1996).

[8] Martinez, J., Reca, J., Morillas, M.T. and Lopez, J.G. “Design and calibration of a compound sharp-crested weir", Journal of Hydraulic Engineering, 131(2), pp. 112-116 (2005).

[9] Ramamurthy, A.S., Tadayon, R. and Chen, Z. "Numerical simulation of sharp-crested weir flows", Canadian Journal of Civil Engineering, 36(9), pp. 1530-1534 (2009).

[10] Afzalimehr, H. and Bagheri, S. "Discharge coefficient of sharp-crested weirs using potential flow", Journal of Hydraulic Research, 47(6), pp. 820-823 (2009).

[11] Nehari, Z. “Conformal mapping”, Mc-Graw- Hill, New York (1952).

[12] Elcrat, A.R. and Trefethen, L.N. "Ideal jet flow in two dimensions", Journal of Fluid Mechanics, 185, pp. 275-288 (1987).

[13] Abd-el-Malek, M.N., Hanna, S.N. and Abd-el-Malek, M.B. "Approximate solution for gravity flow under a Tainer gate", Journal of Computational and Applied Mathematics, 26(3), pp. 271-279 (1989).

[14] Abd-el-Malek, M.B. and Hanna, S.N. "Approximate solution of a flow over a ramp for large Froud number", Journal of Computational and Applied Mathematics, 28, pp. 105-117 (1989).

[15] Abd-el-Malek, M.B. "Approximate solution of gravityaffected flow from planar sluice gate at high Froude number", Journal of Computational and Applied Mathematics, 35, pp. 83-97 (1991).

[16] Guo, J. and Jilien, P.Y. "Shear stress in smooth rectangular open-channel flows", Journal of Hydraulic Engineering, 
131(1), pp. 30-37 (2005).

[17] Belaud, G. and Litrico, X. "Closed-form solution of the potential flow in a contracted flume", Journal of Fluid Mechanics, 599, pp. 299-307 (2008).
[18] Lamb, H. "Hydrodynamics", $6^{\text {th }}$ edition, Cambridge University Press, Dover, New York (1993).

[19] Kabiri-Samani, A.R. "Analytical approach for flow over an Oblique weir”, Scientia Iranica, 17(2), pp. 107-117 (2010). 